\title{
IMMUNOPROTECTION OF SOLUBLE EGG ANTIGENS AND PRAZIQUANTEL IN CHALLENGED MURINE SCHISTOSOMIASIS MANSONI \\ By
}

\author{
NAGLAA F. ABD EL-AAL ${ }^{1 *}$, FATEN A. MOHAMMED ${ }^{1}$, AND MONA MAGDY ${ }^{2}$
}

Department of Medical Parasitology, Faculty of Medicine, Zagazig University ${ }^{1}$, and Department of Pathology, Theodor Bilharz Research Institute ${ }^{2}$, Imbaba B.O.P. 30, Giza, Egypt (*Correspondence:naglaa_fathy220@yahoo.com)

\begin{abstract}
Control of schistosomiasis would be greatly improved by the development of a vaccine, which could be combined with chemotherapy. The current study evaluated the potential effect of soluble egg antigens (SEA) associated with or without PZQ in Schistosoma mansoni challenged mice through parasitological, histopathological and serological studies. Sixty Swiss albino female mice divided into 5 groups (12 mice each); each group was subdivided into 2 groups (6 mice each) according initial and challenged infections. Mice were sacrificed by rapid decapitation 8 weeks post initial and challenge infections for assessment. The current results showed marked reduction of egg counts per gram stool, worm burden, granuloma number and size in combined SEA and PZQ group. Also, significant increase in serum IL-12 \& INF- $\gamma$, plus marked decrease in serum IL-4 during initial and challenged infections. The role of PZQ alone was mainly during primary infection and had no role in reinfection, whereas, SEA alone was effective during challenged one. In conclusion, from the obtained results of the current study the combination of SEA \& PZQ was as a possible potent and promising prophylactic treatment for schistosomiasis especially during re-infection in endemic areas. This prophylactic effect was significantly greater than those observed in the single drug treatment or vaccine used.
\end{abstract}

Keywords: Schistosomiasis, Soluble Egg Antigen, Cytokines, Vaccine, Praziquantel, IL-12, INF $\gamma$, IL-4.

\section{Introduction}

Schistosomiasis is the third tropical disease in the world, following malaria and intestinal helminthiasis; that cause significant morbidity and mortality (WHO, 2016).

Praziquantel (PZQ) is the drug of choice for treating all species of schistosomes, because of its efficacy, ease of administration, safety, and low cost which enhances immune responses to crude schistosome antigens (Wang et al, 2012). However, PZQ kills only adult Schistosoma worms, not juvenile stages; also, is unable to prevent reinfections in humans and does not have prophylactic effects. Hence, there is an urgent demand for novel vaccines and strategies for the prevention of schistosomiasis as well as related reinfections. Improved helminth control is required to improve the global burden of schistosomiasis and its pathologies (Yepes et al, 2015).

Immunization against schistoromiasis using soluble egg antigens (SEA) were studied in mice with the initiation of variable levels of protection against infection that causes reduction in worm burden, intestinal egg load and liver pathology (Garcia et al, 2008). Immunization was able to induce more than $67 \%$ protection, but the existence of at least partly protective immunity would make a reasonable complement to drug therapy (Bergquist et al, 2008).

Cytokines production after parasite antigen stimulation reflects a dominant $\mathrm{T}$ helper 1 (Th1) response, with production of interferon- $\gamma$ (IFN- $\gamma$ ) and interleukin-12 (IL-12) (Caldas et al, 2008). Thus, during the acute phase of the disease, the upper hand is for Th1 response, producing elevated levels of Th1 cytokines in the plasma (Pearce and MacDonald, 2002). Then, in the natural progression of the disease, after egg laying, the initial Th1 response is followed by a developing egg antigen-induced regulatory $T$ helper 2 (Th2) response that down regulates the production and effector functions of the 
pro-inflammatory Th1 mediators with accompanying granuloma formation (Anthony et al, 2012).

Schistosomiasis treated with praziquantel resulted in changes in parasite specific humoral and cellular immune responses. This change is believed to be due to an increase in the amount of antigen exposed to the immunity by treatment and a removal of immunosuppressive effects of mature worm (Mutapi, 2001). Some of the changes occurring following treatment have been associated with resistance to infection or re-infection (Bourke et al, 2014). Schistosomiasis treated with PZQ complemented with immunization resulted in significant reduction of worm burden and rise of specific immunoglobulins potentiated an anti-pathology effect that minimized and ameliorated liver (Teixeira de Melo et al, 2010). The combination of protection using SEA and treatment was recommended in several studies as it provided many complementary goals, a reduction of egg induced pathology, minimal parenchymal changes and the eradication of worms (Rabia et al, 2010). Immunization with recombinant tegument nucleotidases associated with praziquantel treatment was the most immunogenic than each alone. It induced the highest antibody levels, mainly IgG1, associated with high TNF- $\alpha$ and a Th17 (Rofatto et al, 2013).

The combination of PZQ and SEA vaccinated Schistosoma mansoni infected mice enhance the cytokine patterns, reduction of egg induced pathology and eradication of worms thus prevention of reinfection. The current study aimed to evaluate the potential effect of soluble egg antigen (SEA) associated with or without PZQ in Schistosoma mansoni challenged mice through parasitological, histopathological and serological studies (cytokine patterns).

\section{Materials and Methods}

Study type: Non randomized control trial experimental study was performed at the laboratories of Parasitology Department, Faculty of Medicine, Zagazig University and
Theodor Bilharz Research Institute (TBRI) from November 2015 to July 2016. Cercariae of $S$. mansoni (Egyptian strain) were obtained from Biomphalaria alexandrina experimentally infected snails, provided by Schistosome Biological Supply Program Unit (SBSP), TBRI.

Sixty laboratory bred Swiss albino female mice (aged 8 to 10 weeks and weighing 18 to $20 \mathrm{~g}$ each) were used in this study. Mice were fed on standard diet with free accessibility to water at SBSP Unit, TBRI, Giza, Egypt.

Infection: Mice were initially infected with \pm 100 S. mansoni cercariae by tail immersion method (Liang et al, 1987) and were challenged with $\pm 100 S$. mansoni cercariae by the tail immersion method 8 weeks post initial infection.

In vaccinated groups, infection was done 2 weeks after the last dose of vaccination.

SEA antigens preparation and administration (Boros and Warren, 1970): soluble egg antigen (SEA) was prepared at the SBSP Unit, TBRI. The total protein content of SEA preparation was determined according to Bradford (1976). The vaccination schedule was performed (Nabih and Soliman, 1986). Each mouse was injected subcutaneously by $200 \mu 1$ of SEA as the initial dose with total antigen concentration contained $30 \mu \mathrm{g}$ proteins. A second subcutaneous injection (booster dose) of $200 \mu \mathrm{l}$ of the same antigen was given after two weeks containing $20 \mu \mathrm{g}$ proteins; hence, each mouse received a total antigen dose of $50 \mu \mathrm{g}$ protein.

Praziquantel (PZQ) used was distocide (EPICO, Egypt). The drug was given in the form of aqueous suspension (in 3\% ethanol and $7 \%$ Tween 80 at a concentration of 40 $\mathrm{g} / \mathrm{L}), 500 \mathrm{mg} / \mathrm{kg}$ single oral dose 6 weeks post initial infection by means of polythene tube fitted to a tuberculin syringe.

Experimental design: Sixty Swiss albino female mice divided into 5 groups (12 mice each); each group was subdivided into two groups (6 mice each) according initial (1) and challenged infections (Tab. 1) Control hea- 
lthy (GC) uninfected unvaccinated untreated control, subdivided into $\mathrm{C}_{1} \& \mathrm{C}_{2}$. Control infected (GCI) S. mansoni infected unvaccinated untreated; subdivided into $\mathrm{CI}_{1} \& \mathrm{CI}_{2}$. Infected Treated (GIT) infected mice then treated with PZQ $(500 \mathrm{mg} / \mathrm{kg})$; subdivided into $\mathrm{GIT}_{1} \& \mathrm{GIT}_{2}$. Vaccinated Infected $\left(\mathrm{GVI}_{1}\right)$ vaccinated with SEA (50ug) then infected subdivided into $\mathrm{VI}_{1} \& \mathrm{VI}_{2}$. Vaccinated Infected Treated (VIT) vaccinated with SEA (50ug), infected then treated with PZQ $(500 \mathrm{mg} / \mathrm{kg})$ subdivided into $\mathrm{VIT}_{1} \&$ $\mathrm{VIT}_{2}$.

Study assessment: Mice were sacrificed by rapid decapitation 8 weeks post initial and challenge infections for assessment by parasitological and histopathological and serological studies.

Parasitologically: Egg count per gram stool was done by Kato thick smear method (Katz et al, 1972) for each mouse started $6^{\text {th }} \mathrm{W}$ post infection to ensure establishment of infection and as a follow up. Worm burden perfusion mature adult worms from the liver and porto-mesenteric system was performed 8 weeks after infection (Duvall and Dewitt, 1967). The degree of protection (\% reduction of worms) was calculated as follows: $\mathrm{P}$ $(\% \mathrm{R})=\mathrm{C}-\mathrm{T} / \mathrm{C} \times 100 . \mathrm{P}=$ the percentage protection, $\mathrm{C}=$ the mean number of adults recovered from infected mice and $\mathrm{T}$ was the mean of adults recovered from tested mice groups (Tendler et al, 1986).

Histopathologically: The liver was removed from each one of the sacrificed mice, rinsed with phosphate-buffered saline, and fixed in Bouin fluid for $4 \mathrm{~h}$, and then transferred to $70 \%$ alcohol for several days. After dehydration in absolute alcohol and clearing in xylol, it was embedded in paraffin wax, and sectioned at a thickness of $5 \mu \mathrm{m}$. Sections were stained with hematoxylin and eosin (H\&E), and examined for peri-ocular granulomas. The diameter of granulomas was measured using an ocular micrometer. This was done only for granulomas containing ova in their centers and not for confluent ones. The mean diameter for each group was calculated. Granuloma count was determined in five successive fields of magnification, from serial tissue sections more than $25 \mu \mathrm{m}$ apart (Von Lichtenberg, 1962). The percent reduction in granuloma diameter relative to infected control was calculated as follows: \% reduction of granuloma diameter $=$ mean diameter of controls - mean diameter of tested groups / mean diameter of control group x 100.

Serologically: Cytokine measurements: Serum levels of IL-12, IFN- $\gamma$ and IL-4 (BioSource International, Inc., Camarillo, California, USA) were estimated by enzyme linked immunosorbent assays (ELISA) according to the manufacturer's instructions. These assays employ the quantitative sandwich enzyme immune assays. Optical density (OD) values were measured at $450 \mathrm{~nm}$ and serum IL-12, IFN- $\gamma$ and IL-4 concentrations determined from the standard curves. The sensitivity cut-off values for each of cytokines were IL-12 (15pg/ml), IFN- $\gamma(20 \mathrm{pg} /$ $\mathrm{ml})$ and IL-4 (12 pg/ml).

Animal ethics: Experimental animals were used in this study according to international guidelines approved by the Ethics Committee for Animal Experimentation, Faculty of Medicine, Zagazig University.

Statistical analysis: Data checked and analyzed using SPSS version 19 windows and expressed as mean \pm standard deviation (SD). The statistical significance of differential data between experimental groups and controls was determined by an unpaired Student's $t$ test. $\mathrm{P}<0.05$ was considered significant and $\mathrm{p}<0.001$ was considered highly significant.

\section{Results}

The mean egg count for eggs per gram stools (EPG) and \% of their reduction showed no significant difference between GIT and GCI during challenged infection, with slight decrease $(p<0.05)$ during initial one. On the other hand, GVI showed high significant difference $(p<0.001)$ compared to GCI during challenged infection, with slight decrease during initial one $(p<0.05)$. GVIT 
showed the highest significant difference $(p<0.001)$ compared to CI with more reduction in GVIT compared to GVI during initial and challenged infections with more reduction during challenged one (Tab. 2).

The total number of worms and the percent reduction of worm burden showed high significant difference $(p<0.01)$ between and GIT and GCI during initial infection while this reduction decreased during challenged one $(p<0.05)$. Whereas, GVI showed high significant difference $(p<0.001)$ compared to GCI during challenged infection, with slight decrease during initial one $(p<0.05)$. But, GVIT showed the highest significant difference $(p<0.001)$ compared to GCI during initial and challenged infections with more reduction during challenged one (Tab.3).

Granuloma number in GIT showed no significant difference compared to GCI during challenged infection, but with significant reduction during initial one $(\mathrm{p}<0.05)$. On the opposite, GVI showed no significant difference compared to GCI during initial infection, but with significant reduction during challenged one $(p<0.05)$. On the other hand, GVIT showed highest significant difference $(p<0.001)$ compared to GCI during initial and challenged infections with more reduction during challenged one (Tab. 4; Fig. 1). Although granuloma diameter showed slight decrease in GIT compared to GCI $(p<0.05)$ during initial infection, there was no significant difference during challenged one. On the other hand, GVI showed significant difference $(p<0.05)$ compared to GCI during initial and challenged infections with more reduction during challenged one. While, GVIT showed highest significant difference $(p<0.001)$ compared to GCI during initial and challenged infections with more reduction during challenged one (Tab. 5; Fig. 1).

Serum cytokines: The profile of Th-1 related serum cytokine IL-12 showed insignificant increase in GIC $(\mathrm{P}>0.05)$ compared to GC. On the other hand it showed slightly significant increase in GIT \& GVI $(\mathrm{P}<0.05)$, with highest significant increase $(p<0.001)$ in GVIT compared to GCI during initial infection. Whereas, during challenge infection there insignificant increase in in GCI and GIT $(\mathrm{P}>0.05)$ compared to GC. On the other hand, it showed slightly significant increase in GVI and highest significant increase $(p<0.001)$ in GVIT compared to GC and GCI. Also, the profile of Th-1 related serum cytokine IFN- $\gamma$ showed slightly significant increase in GCI and GVI $(\mathrm{P}<0.05)$ compared to GC. On the other hand it showed highest significant increase $(p<0.001)$ in GIT and GVIT compared to GC and slightly significant increase in GIT and highest significant increase in GVIT compared to GCI during initial infection. While during challenged infection serum INF- $\gamma$ showed insignificant increase in GCI ( $>00.05)$ compared to GC, with slightly significant increase $(\mathrm{p}<0.05)$ in GIT \& GVI and highest significant increase $(p<0.001)$ in GVIT compared to GC. In addition to, insignificant increase $(p<0.05)$ in GIT, with slightly significant increase $(p<0.05)$ in GVI group and highest significant increase $(p<0.001)$ in GVIT compared to GIC (Tab. 6). The profile of Th-2 related serum cytokine IL-4 showed high significant increase $(p<0.001)$ in GCI, but slightly significant increase $(p<0.05)$ in GIT, GVI and GVIT compared to GC. Also, serum IL-4 showed insignificant increase $(p>0.05)$ in GIT, with slight significant increase in GVI $(p<0.05)$ and highest significant increase in GVIT $(\mathrm{P}<0.001)$ compared to GCI during initial infection. But, during challenge infection serum IL-4 showed high significant increase $(p<0.001)$ in GIC, slightly significant increase $(p<0.05)$ in IT and insignificant increase $(p>0.05)$ in VI \& GVIT compared to $\mathrm{GC}$, also, insignificant increase $(\mathrm{p}>0.05)$ in GIT, with slightly significant increase $(\mathrm{p}<$ 0.05 ) in GVI and highest significant increase $(\mathrm{P}<0.001)$ in GVIT compared to GCI (Tab. $6)$. 
Table 1: Experimental plan

\begin{tabular}{|c|c|c|c|c|c|c|c|c|c|c|}
\hline \multirow{3}{*}{$\begin{array}{c}\text { Groups } \\
\mathrm{G}_{1}\end{array}$} & \multicolumn{10}{|c|}{ Weeks (wks) ofinitial (1ry) infection } \\
\hline & \multicolumn{4}{|c|}{ Weeks of vaccination } & \multicolumn{4}{|c|}{ Weeks of infection } & \multirow{2}{*}{\multicolumn{2}{|c|}{$8 \mathrm{wks}$}} \\
\hline & $1 \mathrm{wk}$ & 2wks & $3 \mathrm{wks}$ & 4wks & $1 \mathrm{wks}$ & $2-5 \mathrm{wks}$ & 6wks & 7wks & & \\
\hline $\mathrm{C}_{1}$ & & & & & & & & & \multicolumn{2}{|c|}{ sacrificed } \\
\hline $\mathrm{CI}_{1}$ & & & & & I & & & & \multicolumn{2}{|c|}{ sacrificed } \\
\hline $\mathrm{IT}_{1}$ & & & & & $\mathrm{I}$ & & $\mathrm{T}$ & & \multicolumn{2}{|c|}{ sacrificed } \\
\hline $\mathrm{VI}_{1}$ & $\mathrm{~V}$ & & $\mathrm{~V}$ & & $\mathrm{I}$ & & & & \multicolumn{2}{|c|}{ sacrificed } \\
\hline $\mathrm{VIT}_{1}$ & $\mathrm{~V}$ & & $\mathrm{~V}$ & & $\mathrm{I}$ & & $\mathrm{T}$ & & \multicolumn{2}{|c|}{ sacrificed } \\
\hline Groups & \multicolumn{10}{|c|}{ Weeks (wks) of challenged infection } \\
\hline $\mathrm{G}_{2}$ & $1 \mathrm{wk}$ & $2 \mathrm{wks}$ & 3 wks & 4wks & $1 \mathrm{wk}$ & $2-5 \mathrm{wks}$ & $6 \mathrm{wks}$ & $7 \mathrm{wks}$ & $8 \mathrm{wks}$ & $16 \mathrm{wks}$ \\
\hline $\mathrm{C}_{2}$ & & & & & & & & & & sacrificed \\
\hline $\mathrm{CI}_{2}$ & & & & & $\mathrm{I}$ & & & & $\mathrm{Ch}$ & sacrificed \\
\hline $\mathrm{IT}_{2}$ & & & & & $\mathrm{I}$ & & $\mathrm{T}$ & & $\mathrm{Ch}$ & sacrificed \\
\hline $\mathrm{VI}_{2}$ & $\mathrm{~V}$ & & $\mathrm{~V}$ & & $\mathrm{I}$ & & & & $\mathrm{Ch}$ & sacrificed \\
\hline $\mathrm{VIT}_{2}$ & $\mathrm{~V}$ & & $\mathrm{~V}$ & & $\mathrm{I}$ & & $\mathrm{T}$ & & $\mathrm{Ch}$ & sacrificed \\
\hline
\end{tabular}

$\mathrm{C}$ control healthy; I infected; CI control infected; T treated; IT infected treated; V vaccinated; VI vaccinated infected; VIT vaccinated, infected, treated; Ch challenged.

Table 2: Mean egg count for eggs per gram stools (EPG) and \% reduction during initial \& challenge infections in all groups.

\begin{tabular}{|c|c|c|c|c|}
\multirow{2}{*}{$\begin{array}{c}\text { parameters } \\
\text { Groups }\end{array}$} & egg count/g stools during initial infection $\left(_{1}\right)$ & egg count/g stools during challenge infection $\left({ }_{2}\right)$ \\
\cline { 2 - 5 } & Mean \pm SD & \%Reduction & Mean \pm SD & \%Reduction \\
\hline C & - & -- & - & -- \\
\hline CI & $563.3 \pm 34.2$ & - & $512.5 \pm 23.8$ & -- \\
\hline IT & $132.6 \pm 31.9^{*}$ & $76.5 \%$ & $498.2 \pm 26.4$ & $2.8 \%$ \\
\hline VI & $242.8 \pm 32.6^{*}$ & $56.9 \%$ & $114.3 \pm 26.3 *$ & $77.7 \%$ \\
\hline VIT & $116.7 \pm 19.7 * *$ & $79.3 \%$ & $19.4 \pm 22.4^{* *}$ & $96 \%$ \\
\hline
\end{tabular}

*Significant difference compared to infected control $(\mathrm{P}<0.05)$. ${ }^{* *}$ Highly significant difference in compared to infected control $(\mathrm{P}<0.001)$.

Table 3: Worm burden and \% of reduction during initial \& challenge infections in all groups.

\begin{tabular}{|c|c|c|c|c|}
\hline \multirow{2}{*}{ Parameters } & \multicolumn{2}{|c|}{ Worm burden during initial infection $\left(_{1}\right)$} & \multicolumn{2}{c|}{ Worm burden during challenge infection $\left({ }_{2}\right)$} \\
\cline { 2 - 5 } & Mean \pm SD & \%Reduction & Mean \pm SD & \% Reduction \\
\hline C & - & - & - & -- \\
\hline CI & $66.5 \pm 23.8$ & - & $98.4 \pm 26.2$ & -- \\
\hline IT & $17.2 \pm 26.4^{* *}$ & $74.1 \%$ & $72.6 \pm 16.6^{*}$ & $26.2 \%$ \\
\hline VI & $32.3 \pm 26.3^{*}$ & $51.4 \%$ & $19.2 \pm 13.7^{* *}$ & $80.5 \%$ \\
\hline VIT & $13.4 \pm 22.4^{* *}$ & $79.9 \%$ & $1.8 \pm 8.6^{* *}$ & $98.2 \%$ \\
\hline
\end{tabular}

*Significant difference compared to infected control $(\mathrm{P}<0.05)$. **Highly significant difference in compared to infected control $(\mathrm{P}<0.001)$.

Table 4: Mean number of hepatic granulomas and \%reduction during initial \& challenge infections in control \& tested groups.

\begin{tabular}{|c|c|c|c|c|}
\hline \multirow{2}{*}{$\begin{array}{c}\text { Parameters } \\
\text { Groups }\end{array}$} & Hepatic granuloma number during 1ry infection $\left(_{1}\right)$ & \multicolumn{2}{|c|}{ Hepatic granuloma number during challenge infection $\left(_{2}\right)$} \\
\cline { 2 - 5 } & Mean \pm SD & \%Reduction & Mean \pm SD & \%Reduction \\
\hline C & - & -- & - & -- \\
\hline CI & $17.2 \pm 12.6$ & - & $22.3 \pm 12.2$ & - \\
\hline IT & $11.6 \pm 14.9 *$ & $32.6 \%$ & $19.6 \pm 14.9$ & $12.1 \%$ \\
\hline VI & $13.3 \pm 13.4$ & $22.7 \%$ & $10.4 \pm 18.6^{*}$ & $53.4 \%$ \\
\hline VIT & $7.8 \pm 10.7 * *$ & $54.7 \%$ & $3.5 \pm 13.7 * *$ & $84.3 \%$ \\
\hline
\end{tabular}

*Significant difference compared to infected control $(\mathrm{P}<0.05)$. ${ }^{* *}$ Highly significant difference in compared to infected control $(\mathrm{P}<0.001)$.

Table 5: Mean diameter of hepatic granulomas (um) and \%reduction during initial $\left(_{1}\right) \&$ challenge $(2)$ infections in control and tested groups

\begin{tabular}{|c|c|c|c|c|}
\hline \multirow{2}{*}{ parameters } & \multicolumn{2}{|c|}{$\begin{array}{c}\text { Hepatic granuloma diameter (um) during initial } \\
\text { infection }\left({ }_{1}\right)\end{array}$} & \multicolumn{2}{|c|}{$\begin{array}{l}\text { Hepatic granuloma diameter (um) during challenge } \\
\text { infection }\left({ }_{2}\right)\end{array}$} \\
\hline & Mean \pm SD & \%Reduction & Mean \pm SD & $\%$ Reduction \\
\hline $\mathrm{C}$ & - & -- & - & -- \\
\hline $\mathrm{CI}$ & $314.5 \pm 21.8$ & & $332.5 \pm 11.2$ & -- \\
\hline IT & $215.2 \pm 16.2 *$ & $31.6 \%$ & $306.8 \pm 21.3$ & $7.7 \%$ \\
\hline VI & $258.3 \pm 14.3 *$ & $17.9 \%$ & $166.2 \pm 26.6^{*}$ & $50 \%$ \\
\hline VIT & $148.4 \pm 13.6 * *$ & $52.8 \%$ & $58.4 \pm 18.5 * *$ & $82.4 \%$ \\
\hline
\end{tabular}


Table 6: Serum cytokine levels (pg/ml) during initial $\left(_{1}\right)$ \& challenge $\left(_{2}\right)$ infections in control and tested groups.

\begin{tabular}{|c|c|c|c|c|c|c|}
\hline \multirow{2}{*}{ Groups } & \multicolumn{2}{|c|}{ IL-12 (pg/ml) } & \multicolumn{2}{|c|}{ INF- $\gamma(\mathrm{pg} / \mathrm{ml})$} & \multicolumn{2}{|c|}{ IL-4 (pg/ml) } \\
\hline & $\begin{array}{c}\text { 1ry infection } \\
\left(\mathrm{G}_{1}\right)\end{array}$ & $\begin{array}{c}\text { Ch infection } \\
\left(\mathrm{G}_{2}\right)\end{array}$ & $\begin{array}{l}\text { 1ry infection } \\
\left(\mathrm{G}_{1}\right)\end{array}$ & $\begin{array}{c}\text { Ch infection } \\
\left(\mathrm{G}_{2}\right)\end{array}$ & $\begin{array}{c}\text { 1ry infection } \\
\left(\mathrm{G}_{1}\right)\end{array}$ & $\begin{array}{c}\text { Ch infection } \\
\left(\mathrm{G}_{2}\right)\end{array}$ \\
\hline $\mathrm{C}$ & \multicolumn{2}{|c|}{$198.3 \pm 26.2$} & \multicolumn{2}{|c|}{$240.7 \pm 23.4$} & \multicolumn{2}{|c|}{$16.4 \pm 0.54$} \\
\hline CI & $282.7 \pm 23.6$ & $235.4 \pm 12.3$ & $421.3 \pm 18.3^{\mathrm{a}}$ & $320.2 \pm 11.4$ & $73.2 \pm 12.6^{\mathrm{A}}$ & $89.7 \pm 16.3^{\mathrm{A}}$ \\
\hline IT & $474.3 \pm 20.4^{\mathrm{a}, \mathrm{b}}$ & $294.3 \pm 10.6$ & $592.2 \pm 14.2^{\mathrm{A}, \mathrm{b}}$ & $394.4 \pm 10.3^{\mathrm{a}}$ & $46.3 \pm 16.4^{\mathrm{a}}$ & $68.6 \pm 12.7^{\mathrm{a}}$ \\
\hline VI & $352.9 \pm 17.8^{\mathrm{a}}$ & $385.2 \pm 13.6^{\mathrm{a}, \mathrm{b}}$ & $460.6 \pm 12.7^{\mathrm{a}}$ & $430.5 \pm 9.2^{\mathrm{a}, \mathrm{b}}$ & $55.6 \pm 11.8^{\mathrm{a}, \mathrm{b}}$ & $34.9 \pm 18.2^{b}$ \\
\hline VIT & $690.8 \pm 16.3^{\mathrm{A}, \mathrm{B}}$ & $548.7 \pm 8.9^{\mathrm{A}, \mathrm{B}}$ & $864.4 \pm 13.8^{\mathrm{A}, \mathrm{B}}$ & $658.3 \pm 8.6^{\mathrm{A}, \mathrm{B}}$ & $28.7 \pm 14.2^{\mathrm{a}, \mathrm{B}}$ & $19.8 \pm 13.4^{\mathrm{B}}$ \\
\hline
\end{tabular}

${ }^{\mathrm{a}}(\mathrm{P}<0.05),{ }^{\mathrm{A}}(\mathrm{P}<0.001)$ Significant difference compared to healthy control. ${ }^{\mathrm{b}}(\mathrm{P}<0.05),{ }^{\mathrm{B}}(\mathrm{P}<0.001)$ Significant difference compared to infected control.

\section{Discussion}

There is a clear reduction in the morbidity during a reinfection exposure to human $S$. mansoni (Savioli et al, 1997). Likewise, there is a reduction in lesion severity in a secondary exposure following chemotherapy in mice (Coelho et al, 1996) and baboons (Nyindo et al, 1999). The reinfection exposure next chemotherapy led to ameliorated granulomatous inflammation in mice. Furthermore, depending on the mode of primary cercarial exposure, the reduction might be associated with either a Th1 or a Th2 immune response. This possibility of modulating the immune response through modification of primary infection procedures may be of significant benefit in the optimization of vaccine programmes (Farah et al, 2000).

It was trusted that $S$. mansoni infections induce dominated Th2 cells immune response, while a Th1 predominant response powerfully associates with resistance to infection (Jankovic et al, 1999). Typically, Th1 responses are categorized by the secretion of IL-12, IFN- $\gamma$ and IL-2. Though, IL-4, IL-5, IL-6, and IL-10 are cytokines specific to Th2 responses (Romeih et al, 2008).

The present study corroborated these findings and showed that the characteristic increase of schistosome egg-induced Th2associated cytokines during the acute phase of a primary infection (Wynn and Cheever, 1995) was clearly lacking in a reinfection exposure. Also, the results showed that the profile of cytokines during the reinfection period was affected by the pattern of previous treatment. Thus, on re-infection, mice previously infected and then treated with praziquantel developed a Th1-dominant re- sponse with elevated SEA-induced IFN- $\gamma$. Nonetheless, PZQ alone was unlikely to reduce challenged infection for this reason; we used SEA alone and in combination with PZQ to verify their effects on exposure to reinfection. This agreed with Abdel-Ghaffar et al. (2005) who found reported that the combination of protection using SEA and PZQ was recommended as it provided various complementary goals, a reduction of egg induced pathology, minimal parenchymal changes and eradication of worms. Therefore, evaluation the treated, immunized infected mice was important by studying several criteria related to the parasitic intensity, stages and distribution through the host tissues.

In this study, SEA was used since, SEA contains a range of antigens that cross-react with cercariae antigen, adult worm antigen, and schistosomules (Curwen et al, 2004), and thus increased reactivity to egg-stage parasites may promote anti-larval and antiadult worm responses (Woolhouse, 1994).

In the present study, Kato-Katz method was the cornerstone for diagnosis of S. mansoni infection, with a simple inexpensive procedure, and widespread use in endemic areas of S. mansoni (Teixeira et al, 2007). Kato-Katz method was used from the $6^{\text {th }} \mathrm{w}$ post infection to ensure infection and follow up. The results showed the greatest reduction in egg count/gram stool was in GVIT during initial and challenged infections with more reduction during challenged one, indicating that PZQ and SEA combination was protective especially on exposure to reinfection more than each one alone. Also the role of PZQ alone was mainly during primary 
infection and had no role in reinfection, while SEA alone had slight role during initial and challenged infections.

In the current work, the greatest reduction in worm burden was in GVIT during initial and challenged infections with more reduction during challenged one, indicating that PZQ and SEA combination was protective especially on exposure to reinfection more than each one alone. Also, the role of PZQ alone was mainly during primary infection and had no role in reinfection, while SEA alone had no role during initial infection and slight role during challenged one.

These results went hand to hand to Suleiman et al. (2004) and Rabia et al. (2010). In addition, Yepes et al. (2015) suggested a significant reduction in adult worm burden followed the combined treatment of PZQ and edelfosine as a potential vaccine.

The death of the worms due to the treatment with antischistosomal drugs was attributed to metabolic disorders, mechanical destruction and muscular contraction of the treated worms (Doenhoff et al, 2002). The parasitological improvement is due to antiparasitic drug PZQ which causes direct or indirect toxic effect in combination with the effect of immunization with SEA which lead marked decrease in the worm number or fecundity due to hindering the process of oviposition (Guirguis, 2003).

The granuloma formation and fibrosis are the key factors of morbidity and mortality in schistosomiasis. Although the granuloma formation is helpful for the host because it blocked the hepatotoxic effect of antigen released from parasite eggs, this process might lead to the fibrosis with excessive accumulation of collagen and other extracellular matrix proteins in the periportal space (Henri et al, 2002). The manifestations of schistosomiasis are mainly attributed to granulomatous inflammation around parasite eggs (Abath et al, 2006). The formation of granulomas depends predominantly on CD4+ T cell specific for egg antigen and represents a delayed- type hypersensitivity
(Garcia et al, 2008).

In this work, results of granuloma number and diameter was with greatest reduction in the combination of SEA and PZQ during initial and challenged infections with more reduction during challenged one, indicating that PZQ and SEA combination was protective especially on exposure to reinfection more than each one alone. Also the role of PZQ alone was mainly during primary infection and had no role in reinfection, while SEA alone had no or slight role during initial and challenged infections. This may be due to the effect of combined SEA and PZQ that reduced CD4 T cells and increased CD8 cells (El-Ahwany et al, 2006; El-Lakkany and Nosseir, 2007). These results were in agreement with Rabia et al. (2010) and Yepes et al. (2015).

Cytokines are important regulators of immuno-inflammatory responses and play a main role in the regulation of granuloma formation, fibrosis deposition and degradation. Hoffmann et al. (2002) demonstrated the alterations in $\mathrm{T}$ helper cytokine profiles during the formation of granulomatous reaction. In the present study, the levels of cytokines (IL-12, IFN- $\gamma$, IL-4) were measured among all studied groups.

The present result showed that PZQ and SEA alone slightly increased the serum level of Th-1 cytokines; IL-12 and INF- $\gamma$ after it was increased due to $S$. mansoni infection in mice during primary and challenge infections. Also, PZQ was effective mainly during initial infection and the role of SEA appeared mainly during challenged infection. Nevertheless, the greatest effect was with combined PZQ and SEA especially during challenged infection.

These results agreed with Boros (1994) who stated that IFN- $\gamma$ was found to be produced very early at the beginning of the liver granulomatous response, by the time granulomas reached maximal size where its production declined. Caldas et al. (2008) concluded that in the acute phase there was a mixed expression of Th1 and Th2 cytokines 
with predominance of Th1 in the early infection. The higher production of IFN- $\gamma$ in acute schistosomiasis compared to chronic one, partly explain absence of modulation of the immune response in acute infection. These data were similar to de Jesus et al. (2004) who reported that IFN- $\gamma$ is the main Th1 cytokine involved in the down modulation of Th2 cells. Also, Henri et al. (2002) recorded that IFN- $\alpha$ has a major role in the protective immunity against periportal fibrosis. However, Th2- cytokine IL-4 marked increased with Schistosoma infection during initial infection challenged infection; there was slight decrease in serum IL-4 PZQ and SEA alone during initial and challenged infections. On the opposite side, marked reduction in serum IL-4 reported with combined PZQ and SEA especially during challenge infection. This means predominant of Th1 cytokines IL-12 \& INF- $\alpha$ and down regulation of Th2 cytokine IL-4. The present results agreed with Bourke et al. (2014) and Yepes et al. (2015).

During schistosomal infection, both Th1 and $\mathrm{Th} 2$ responded directed against egg antigen and produce IFN- $\gamma$ \& IL-4 respectively (Stadecker et al, 2004). Mice infected with a single large dose of cercariae and then treated with PZQ developed a Th1-dominant response with elevated SEA-induced IFN- $\gamma$ and IL-12. In contrast, animals exposed to repeated small doses in the primary infection mounted a Th2 response with elevated SEAdriven IL-5 when re-infected (Farah et al, 2000).

Praziquantel treatment markedly alters polarization of schistosome-specific cytokine responses, and these changes, particularly in response to egg-stage parasites, may promote resistance to reinfection (Bourke et al, 2013). Praziquental is also known to alter schistosome-specific immune responses, affecting antibody levels and isotype switching (Mutapi, 2001). Given PZQ treatment results in a specific increase in the intensity of the vaccine recognized by serum antibodies (Mutapi et al, 2005). Bourke et al. (2014) reported that the glutathione-S-transferase (GST) -specific cytokine profiles are influenced by boosted PZQ treatment thus influenced cellular responses to a GST vaccine.

The current results strengthen the perspective of combining immunization strategies with chemotherapeutic treatment. The present experiment was designed to allow visualization of the synergistic effect of immunization and chemotherapy on the parasites, through marked reduction of egg counts in stool, worm burden, granuloma number and size and alteration in serum cytokines especially during challenged infection. These results agreed Rofatto et al. (2013).

Generally, Schistosomiasis is a major parasitic disease that affects more than 200 million people worldwide, with more than 600 million people in the tropics are at risk for developing schistosomiasis (CDC, 2015). $S$. mansoni top the list of Egyptian endemic parasitosis (Kilany et al, 2009). The early diagnosis is essential to start treatment, and avoiding the complications of chronic schistosomiasis (Ahmad et al, 2008; Massoud et al, 2010; Ismail et al, 2016). Sabry et al. (2015) reported that their study was one of few, addressing the possibility of direct relation between $S$. mansoni \& hepatic carcinoma, concluding an initial indication of equal risk value of both human chronic $S$. mansoni infection and HCV infections in precipitating hepatocellular carcinoma among Egyptian patients. Schistosomiasis diagnosis requires viable eggs in fecal samples (direct methods). Despite $100 \%$ specificity of these direct parasitological methods, yet their sensitivity is rather poor even with concentration techniques. This may be explained by many factors such as day to day variation, uneven distribution of eggs in the excreta, small amount of excreta examined and light infection. Tissue retaining eggs due to fibrosis is another factor (Venderame et al, 2001). El-Tonsy et al. (2016) stated that Hepatocellular carcinoma (HCC) is one of the most common cancers worldwide, and accounted for about $4.7 \%$ of chronic liver 
disease in Egyptian patients. They concluded that As regards the aggression of $\mathrm{HCC}$, it was more commonly multifocal and larger in size in patients with concomitant infection than in patients with $\mathrm{HCV}$ alone

\section{Conclusions}

The outcome results proved the combination of SEA and PZQ was as a possible potent and promising prophylactic treatment for schistosomiasis. This prophylactic effect was significantly greater than those observed in the single drug treatment or single vaccine used especially during re-infection in endemic areas. The present study recommended to highlight the importance of studying vaccine candidates in different immunization schedules combined with chemotherapeutic agents aiming to select the more appropriate to be employed in human trials.

Conflicts of Interest(s): Authors declared that they neither have conflict of interest nor received financial support.

\section{References}

Abath, FG, Morais, CN, Montenegro, CE, Wynn, TA, Montenegro, SM, 2006: Immunopathogenic mechanisms in schistosomasis: what can be learnt from human studies? Trends Parasitol. 22:85-91.

Abdel-Ghaffar, O, Rawi, SM, Ishaq, AI, 2005: Evaluation of the curative efficacy of RO 15-8843 against mansonian schistosomiasis in albino mice. J. Egypt Ger. Soc. Zool. 47:15-22.

Ahmed, AM, Abdel-Tawab, AH, Morsy, AT A, 2008: Alpha-glutathione S-transferase and serum aminotransferases in schistosomiasis $\mathrm{ma}$ nsoni patients with or without hepatitis $\mathrm{C}$ virus. J. Egypt. Soc. Parasitol. 38, 2:561-72.

Anthony, BJ, Ramm, GA, McManus, DP, 2012: Role of resident liver cells in the pathogenesis of schistosomiasis. Trends Parasitol. 28: 572-9.

Bergquist, R, Utzinger, J, Mc-Manus, DP, 2008: Trick or treat: the role of vaccines in integrated schistosomiasis control. PLoS. Negl. Trop. Dis. 2:244-52.

Boros, DL, 1994: The role of cytokines in the formation of the schistosome egg granuloma. Immunobiol. 191, 4/5:441-50.

Boros, DL, Warren, KS, 1970: Delayed hypersensitivity type III: granuloma formation and dermal reaction induced and elicited by a soluble factor isolated from Schistosoma mansoni eggs. J. Exp. Med. 132, 3:488-507.

Bourke, CD, Nausch, N, Rujeni, N, Appleby, LJ, Mitchell, KM, et al. 2013: Integrated analysis of innate, Th1, Th2, Th17, and regulatory cytokines identifies changes in immune polarization following treatment of human schistosomiasis. J. Infect. Dis. 208:159-69.

Bourke, CD, Nausch, N, Rujeni, N, Appleby, LJ, Trottein, F, et al, 2014: Cytokine responses to the anti-schistosome vaccine candidate antigen glutathione-s-transferase vary with host age and are boosted by praziquantel treatment. PLoS. Negl. Trop. Dis. 8, 5:e2846.

Bradford, MM, 1976: A rapid and sensitive method for the quantitation of microgram qualities of protein utilizing the principle of protein dye binding. Anal. Biochem. 72: 248-54.

Caldas, IR, Campi-Azevedo, AC, Oliveira, L F, Silveira, AM, Oliveira, RC, et al 2008: $\mathrm{Hu}-$ man schistosomiasis mansoni: immune responses during acute and chronic phases of the infection. Acta Trop. 108:109-17.

CDC, 2015: Parasites-Schistosomiasis. Saving Life, Protecting People

Coelho, PMZ, Toppa, NH, Feldman, JS, Goncalves, R, Mello, RT, 1996: Schistosoma mansoni: Permanence of modulation of the granulomatous inflammatory response in mice cured in the chronic phase. Int. J. Parasitol. 26:139395.

Curwen, RS, Ashton, PD, Johnston, DA, Wilson, RA, 2004: The Schistosoma mansoni soluble proteome: a comparison across four life-cycle stages. Mol. Biochem. Parasitol. 138:57-66.

de Jesus, AR, Magalhaes, A, Miranda, DG, Miranda, RG, Araujo, MI, et al 2004: Association of type 2 cytokines with hepatic fibrosis in human Schistosoma mansoni infection. Infect. Immun. 72, 6:3391-7.

Doenhoff, MJ, Kusel, JR, Coles, GC, Cloll, D, 2002: Resistance of Schistosoma mansoni to praziquantel: is there a problem? Trans. R. Soc. Trop. Med. Hyg. 96:465-69.

Duvall, RH, and Dewitt, WB, 1967: An improved perfusion technique for recovering adult Schistosoma from laboratory animals. Am. J. Trop. Med. Hyg. 16, 4:483-4.

El-Ahwany, EG, Nosseir, MM, Ali IR 2006: Immunonsdulation of pulmonary and hepatic granulomatous response in mice immunized with 
purified lung-stage schistosomulae antigen. J. Egypt. Soc. Parasitol. 36, 2:335-50.

El-Lakkany, N, and Nosseir, M, 2007: Pharcodynamics of pentoxifylline and /or praziquantel in murine schistosomiasis mansoni. APMJS 115: 184-94.

El-Tonsy, MM, Hussein, HM, Helal, Tel-S, Tawfik, RA, Koriem, KM, et al, 2016: Human Schistosomiasis mansoni associated with hepatocellular carcinoma in Egypt: current perspective. J. Parasit. Dis. 40, 3:976-80.

Farah, IO, Johansson, M, Loēvgren-Bengtson, K and Hau, J, 2000: Schistosoma mansoni in mice: the pattern of primary cercarial exposure determines whether a secondary infection post-chemotherapy elicits a $\mathrm{T}$ helper 1 - or a $\mathrm{T}$ helper 2-associated immune response. Scand. J. Immunol. 51:237-43.

Garcia, TC, Fonseca, CT, Pacifico, LG, Duraes, Fdo, V, Marinho, FA, et al 2008: Peptides containing $\mathrm{T}$ cell epitopes, derived from Sm 14, but not from paramyosin, induce a Th1 type of immune response, reduction in liver pathology and partial protection against Schistosoma mansoni infection in mice. Acta. Trop. 106, 3:162-7.

Guirguis, FR, 2003: Efficacy of praziquantel and Ro 15-5458, a qacridanonchydrazone derivative, against Schistosoma haematobium. Arzeim Forsch Drug Res. 53, 1:57-61.

Henri, S, Chevillard, C, Mergani, A, Paris, P, Gaudart J, et al 2002: Cytokine regulation of periportal fibrosis in humans infected with Schistosoma: IFN-gamma is associated with protection against fibrosis and TNF- $\alpha$ with aggravation of disease. J. Immunol. 169, 2:929-36.

Hoffmann, A, Levchenko, A, Scott, ML, Baltimore, D, 2002: The IkappaB-NF-kappaB signaling module: temporal control and selective gene activation. Science 298, 5596:1241-5.

Ismail, HI, Ashour, DS, Abou Rayia, DM, Ali, AL, 2016: Ocular pathological changes in hamsters experimentally infected with Schistosoma mansoni. J. Helminthol. 17:1-6.

Jankovic, D, Wynn, TA, Kullberg, MC, Hieny, S, Caspar, P, et al, 1999: Optimal vaccination against Schistosoma mansoni requires the induction of both B cell- \& IFN-gamma-dependent effector mechanisms. J. Immunol. 162, 1: 345-51.

Katz, N, Chaves, A, Pellegrino, J, 1972: A simple device for quantitative stools thick smear technique in schistosomiasis mansoni. Rev. Inst. Med. Trop. S Paulo 14:397-400.
Kilany, YF, Sahar, A, Abou-Holw, SA, Abouel-Nour, MF, Morsy, ATA, 2009: Early development of osteoporosis in male smokers with hypo and rogenism due to fascioliasis with or without schistosomiasis added by life style $\mathrm{J}$. Egypt. Soc. Parasitol. 39, 3:789-802.

Liang, Y, John, B, Boyed, D, 1987: Laboratory cultivation of schistosome vector snails and maintenance of schistosome life cycles. Proceed. $1^{\text {st }}$ Sino-American Symposium 1:34-48.

Massoud, AM, El Ebiary, FH, Ibrahim, SH, Saleh, HA, Khalil, HH, 2010: Evaluation of "Myrrh extract" against Schistosoma mansoni: a histological study. J. Egypt. Soc. Parasitol. 40, 1: 245-58.

Mutapi, F, 2001: Heterogeneities in anti-schistosome humoral responses following chemotherapy. Trends Parasitol. 17:518-24.

Mutapi, F, Burchmore, R, Mduluza, T, Foucher, A, Harcus, Y, et al, 2005: Praziquantel treatment of individuals exposed to $S$. haematobium enhances serological recognition of defined parasite antigens. J. Infect. Dis. 192, 6:110-8.

Nabih, I, Soliman, AM, 1986: Studies on fresh water snails, specific intermediate host for schistosomiasis. II. Isolation of total protein from native and irradiated snails. Cell Mol. Biol. 32: 315-7.

Nyindo, M1, Kariuki, TM, Mola, PW, Farah, IO, Elson, L, et al 1999: Role of adult worm antigen-specific immunoglobulin $\mathrm{E}$ in acquired immunity to Schistosoma mansoni infection in baboons. Infect. Immun. 67:636-42.

Pearce, EJ, and MacDonald, AS, 2002: The immunobiology of schistosomiasis. Nat. Rev. Immunol. 2:499-511.

Rabia, I, Nagy, F, Aly, E, Mohamed, A, ElAssal, F, et al, 2010: Effect of treatment with antifibrotic drugs in combination with PZQ in immunized Schistosoma mansoni infected murine model. J. Am. Sci. 6, 5:208-16.

Rofatto, HK, Montoya, BO, Miyasato, PA, Garcia, J, Rodriguez, D, et al, 2013: Immunization with tegument nucleotidases associated with a subcurative praziquantel treatment reduces worm burden following Schistosoma mansoni challenge. Peer J. 1:e58; Doi 10.7717/peerj.58.

Romeih, MH, Hanem, M, Hassan, HM, Abou Shousha, TS, Saber, MA, 2008: Immunization against Egyptian Schistosoma mansoni infection by multivalent DNA vaccine. Acta Biochim. Biophys. Sinica. 40. 4:327-38. 
Sabry, Ael H, El-Aal, AA, Mahmoud, NS, Nabil, Y, Aziz, IZ, 2015: an initial indication of predisposing risk of Schistosoma mansoni infection for hepatocellular carcinoma. J. Egypt. Soc. Parasitol. 45, 2:233-40.

Savioli L, Renganathan E, Montresor A, Davis, A, Behbehani, K, 1997: Control of schistosomiasis. a global picture. Parasitol. Today 13: 444-8.

Stadecker, MJ, Ashai, H, Finger E, Hernandez, HJ, Rutitzky, LI, et al, 2004: The immunobiology of Th1 polarizaiton in high-pathology schistosoniasis. Immunol. Rev. 201:168-79.

Suleiman, MI, Akarim, EI, Ibrahi, KE, Saad, AM, Mohammed, AE, et al, 2004: Antischistosomal effects of praziquantel, its alkaline hydrolysis and sun decomposed products on experimentally $S$. mansoni infected albino mice. (A) Efficacy assessment based on clinicopathological findings. J. Egypt Soc. Parasitol. 34, 1:131-42. Teixeira, CF, Neuhauss, E, Bem, R, Romanzini, J, Graeff-Teixeira, C, 2007: Detection of Schistosoma mansoni eggs in feces through their interaction with paramagnetic beads in a magnetic field. PLoS Negl Trop. Dis. 1:e73.

Teixeira de Melo, T, Michel de Araujo, J, Valle Duraes, FD, Caliari, MV, Oliveira, SC, et al, 2010: Immunization with newly transformed Schistosoma mansoni schistosomula tegument elicits tegument damage, reduction in egg and parasite burden. Parasite Immunol. 32, 11, 12:749-59.

Vendrame, CM, Carvalho, MD, Yamamoto, CR, Nakhle, MC, Carvalho, SA, et al, 2001: Evaluation of anti-Schistosoma mansoni $\mathrm{IgG}$ antibodies in patients with chronic schistosomiasis mansoni before and after specific treatment. Rev. Inst. Med. Trop. Sao Paulo. 43, 3:153-9.

Von Lichtenberg, FC, 1962: Host response to eggs of Schistosoma mansoni. I- Granuloma formation in the sensitized laboratory mouse. Am. J. Pathol. 41:711-31.

Wang W, Wang L, Liang, YS, 2012: Susceptibility or resistance of praziquantel in human schistosomiasis: a review. Parasitol. Res. 111, 5: $1871-7$.

Woolhouse, ME, 1994: Immunoepidemiology of human schistosomes: taking the theory into the field. Parasitol. Today 10, 5:196-202.

WHO, 2016: Schistosomiasis; Available at: http ://www.who.int/mediacentre/factsheets/fs115/en. Wynn, TA, Cheever, AW, 1995: Cytokine regulation of granuloma formation in schistosomiasis. Curr. Opin. Immunol, 7: 505-11.

Yepes, E, Varela-M, RE, López-Abán, J, Rojas-Caraballo, J, Muro, A, et al, 2015: Inhibition of granulomatous inflammation and prophylactic treatment of schistosomiasis with a combination of Edelfosine and Praziquantel. PLoS. Negl. Trop. Dis. 9, 7:e0003893.

\section{Explanation of figures}

Fig. 1: Section of liver-showing normal hepatic architecture of control healthy (C) (H\&E x200).

Fig. 2: Section of liver of control infected (CI) group showing (a) Large number and diameter of Schistosoma mansoni granulomas (cellular, fibrocellular and fibrous) during initial infection (H\&E, X200). (b) Large number and diameter of Schistosoma mansoni granulomas (fibrocellular and fibrous) during challenge infection (H\&E, X200).

Fig. 3: Section of liver of infected treated (IT) showing (a) Decreased number and diameter of Schistosoma mansoni granulomas during initial infection (H\&E, X200). (b) Large number and diameter of Schistosoma mansoni granulomas during challenge infection (H\&E, X200).

Fig. 4: Section of liver of vaccinated infected (VI) showing (a) Large number with slight decreased diameter of Schistosoma mansoni granulomas during initial infection (H\&E, X200). (b) Slight decreased number and diameter of Schistosoma mansoni granulomas during challenge infection (H\&E, X200).

Fig. 5: Section of liver of vaccinated infected treated (VIT) showing (a) Decreased number and diameter of Schistosoma mansoni granulomas during initial infection (H\&E, X200). (b) Large number and diameter of S. mansoni granulomas during challenge infection (H\&E, X200). 


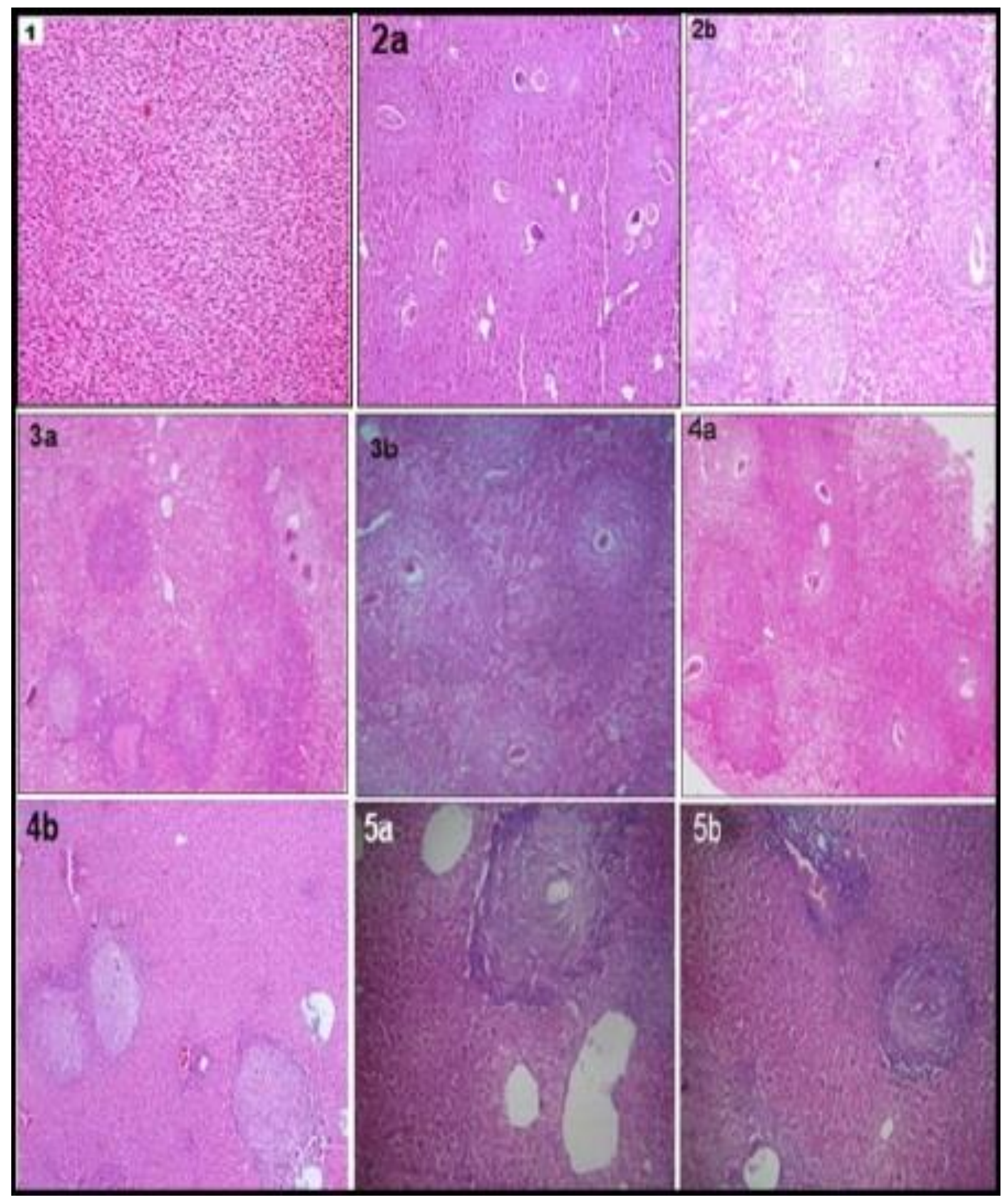

